\title{
Amado Alonso en la Argentina. Una historia global del Instituto de Filologia (1927-1946)
}

MiRANDA LidA (2019).

Bernal, Universidad Nacional de Quilmes, 184 páginas. ISBN 978-987-558-587-4

0 Guiomar E. Ciapuscio

Universidad de Buenos Aires - CONICET, Argentina

\section{Emiliano Battista}

Universidad de Buenos Aires - CONICET, Argentina

\section{Voluntad y azar}

El azar -elemento tan fundamental como la voluntad para trazar destinos- trajo a Amado Alonso a Buenos Aires e hizo de la Argentina su patria de adopción (Guitarte, 1952: 5).

El día 4 de noviembre de 2020 se celebró la presentación del libro de Miranda Lida: Amado Alonso en la Argentina. Una historia global del Instituto de Filología (1927-1946) (2019). Esta reseña es una versión ampliada del texto con que presentamos ese libro. ${ }^{1}$ Su autora, Miranda Lida, es Investigadora Independiente del CONICET y Profesora del Departamento de Humanidades de la Universidad de San Andrés. En su producción científica se pueden identificar dos grandes focos de atención, ambos vinculados con el desarrollo de la actividad política, social y cultural argentina. Por un lado, investigaciones sobre el catolicismo en nuestro país, de las que surgieron, entre otros aportes, las obras Monseñor Miguel De Andrea. Obispo y hombre de mundo (2013) e Historia del catolicismo en la Argentina. Entre el siglo XIX y el XX (2015). Una segunda línea de investigación se ocupa de la reconstrucción histórica y cultural de la labor del Instituto de Filología de la Universidad de Buenos Aires, de la que derivó Años dorados de la cultura. Los hermanos María Rosa y Raimundo Lida y el Instituto de Filología antes del peronismo (2014), emparentado con el material que deviene objeto de esta contribución.

Amado Alonso en la Argentina. Una historia global del Instituto de Filología es un trabajo sumamente interesante, rico en información, muy bien organizado y escrito; es un libro que convoca y provoca al lector, especialmente a los que de una u otra manera están vinculados profesionalmente (y afectivamente) como

1 La intervención inicial estuvo a cargo de Guiomar E. Ciapuscio. Además, contó con los aportes de Ángela Di Tullio y Pablo Buchbinder (https:// www.youtube.com/watch? $\left.v=A x F o D_{4} I F x Z A \& t=9815\right)$. nosotros con el Instituto; pero también interpela desde el ángulo más amplio de la ciudadanía argentina, porque esta es una obra que tiene una manifiesta y potente dimensión histórico-política y cultural. Es una fracción de nuestra historia, marcada-siemprepor un pasado áureo, fulgurante, perdido, cada vez más lejano, y un devenir, en contraste, opaco, sombrío. Ese doloroso contraste se experimenta de manera concentrada y-diríamos-dramática en la historia de ese período del centro de estudios porteño, en paralelo con el tramo vital más sobresaliente de este célebre filólogo. Ambos -esto es, tanto el Instituto como el director en cuestión- con finales abruptos, puesto que Alonso murió prematuramente seis años más tarde de su retiro obligado, en Arlington (Massachusetts, Estados Unidos); como muchos, intuimos que no deberían desligarse completamente ambos desenlaces.

Amado Alonso en la Argentina analiza críticamente el período de emergencia y consolidación del Instituto de Filología, prestando particular atención a su enclave en la cultura argentina y en el mundo hispánico. Sin agotar la investigación en la biografía de Alonso -cuyo nombre actualmente complementa y rubrica la denominación del centro de estudios-, la obra se sirve del derrotero trazado por este versátil hombre de letras: oriundo de Navarra, formado en Madrid bajo el ala de Ramón Menéndez Pidal, especializado en fonética y dialectología en Francia y Alemania, y consagrado en Buenos Aires como figura pública en la materia hasta que, por problemas académico-institucionales derivados de diferencias con el gobierno argentino de entonces, se vio obligado a emigrar a Harvard (Estados Unidos).

El libro tiene el propósito de ofrecer una historia social y cultural del Instituto de Filología desde una perspectiva global, que incluye la perspectiva de la historia de España y de las Américas, la situación de la filología en el panorama occidental y su particular 
inserción en el contexto académico argentino, que no puede de ninguna manera obviar a su protagonista principal: Alonso, quien, en rigor, además de encabezar el título de la obra, es tema fundamental de cuatro de sus cinco capítulos.

El primer capítulo revisa sucintamente la actividad institucional española, tanto en la Península como en América, y su relación con la vida universitaria argentina en los tiempos de la Reforma de 1918. Lida considera que en la disposición a "forjar vínculos atlánticos" de parte de estos organismos está el primer paso para la proyección de un centro de estudios sobre el lenguaje como el finalmente creado en 1922. Formula, pues, la pregunta que funciona como punto de partida: ¿por qué la filología (hispánica)? Aquí nos permitimos ampliarla para organizar mejor el comentario: ¿por qué la necesidad de fundar en Buenos Aires un instituto (argentino) de filología (hispánica) y (con)ceder su gestión a especialistas españoles? Lida responde: en un país que recibía intensas oleadas inmigratorias desde 1880 y que poseía entonces una población cada vez más heterogénea, se requería la presencia de una institución que asumiera un papel público y que tomara posición (científica y cultural) frente al problema de la lengua. La filología era una disciplina que ostentaba rigurosidad de método, capaz de dialogar con las ciencias duras y, por ende, de jerarquizar la literatura, la estética y la filosofía del lenguaje; contaba con una tradición germana forjada durante el siglo XIX que a principios del siglo XX había ingresado a España, donde también se institucionalizó gracias a la labor de Menéndez Pidal, director del Centro de Estudios Históricos (CEH) de Madrid, quien supo "poner de relieve el prestigio de la lengua española en el concierto europeo”. Así, según explica Lida, la creación del Instituto de Buenos Aires fue "fruto de la convergencia de iniciativas variadas": por un lado, los impulsos renovadores asociados al movimiento reformista universitario argentino; por otro, la creciente colaboración de la Universidad porteña con las instituciones españolas -el CEH, la Institución Cultural Española (ICE) y la Junta de Ampliación de Estudios (JAE)-; y, por último, las gestiones de Coriolano Alberini y de Ricardo Rojas, cuyos decanatos en la Facultad de Filosofía y Letras estuvieron signados por una pretensión pedagógica y normativa en cuanto a la lengua, considerada herramienta crucial para acuñar identidades. La creación del centro de estudios porteño, por ende, venía a ampliar sobre nuevas latitudes una red de lazos culturales (transatlánticos) ya dispuesta entre Madrid, Nueva York y San Juan de Puerto Rico.

El segundo capítulo recupera críticamente aquello que describe como la instalación de la filología académica en la Argentina. Lida detecta y analiza la "versatilidad" rápidamente desplegada por este joven (de "prestigio adquirido" e "independencia de criterio") en la arena pública porteña, en la que fue protagonista de las "batallas lingüísticas sobre la cuestión nacional”. Reconstruye también la trama de la polémica filológica en la Argentina a comienzos del siglo XX: las embestidas de Arturo Costa Álvarez y Vicente Rossi contra el Instituto y sus miembros, el enfrentamiento del primero de los mencionados con Alonso, y las interesantísimas disputas libradas entre Américo Castro - primer director del Instituto en 1923-y el joven Jorge Luis Borges. La autora encuentra que, más allá de las riñas discursivas (signadas por recelos académicos que llevaron a personalizar muchos de los comentarios), las sucesivas contiendas en las que intervino Alonso permitieron dejar en claro cuál era su posición respecto del problema de la lengua. Según Lida, el filólogo navarro realizaba un análisis en clave sociológica del habla de los argentinos, en general, y de los habitantes de Buenos Aires, en particular, a quienes distinguía según clases definidas no tanto por su posición socio-económica, sino por sus habilidades lingüístico-culturales. Siguiendo este criterio, e identificando en la inmigración de masas "una de las variables más disruptivas", Alonso no le atribuyó a España una "superioridad ingénita" ni consideró que fuera portadora de los parámetros de "corrección lingüística, a los cuales debían ceñirse los americanos"; no obstante, se pronunciaba en favor de las ventajas de la existencia (legítima) de una "comunidad panhispánica".

El tercer capítulo aborda el período consagratorio del Instituto de Filología, producto del afianzamiento de la gestión de Alonso, quien, según Lida, era un “académico influyente" devenido, pues, en una suerte de "cacique con creciente margen de maniobra" que llegó a manejar "ingentes recursos presupuestarios". Hacia fines de la década de 1930, el Instituto logró invertir la relación de fuerzas originalmente pensada para el centro porteño: en lugar de ser socio menor de la revista editada por Federico de Onís en Puerto Rico con el patrocinio de la Universidad de Columbia, fue esta última la que ingresó como acompañante en el lanzamiento de una de las publicaciones periódicas fundadas en Buenos Aires: la Revista de Filología Hispánica. En esta etapa tuvo lugar también el auge de la producción editorial del Instituto, que gestionaba la publicación de libros especializados en ediciones académicas confeccionadas por la imprenta de la Universidad. La Colección de Estudios Estilísticos (CEE) y la Colección de Teoría y Filosofía del Lenguaje (CFyTL) contenían obras de distribución internacional, bajo un sello distintivo -la editorial Losada- que reunía 
traducciones originales y rigurosas (93). Lida remite al contexto general en el afán de poder dimensionar esta época dorada del centro de estudios porteño y advierte que resultó de la confluencia de diferentes factores: 1) el Estado Argentino reconoció oficialmente la labor del Instituto y le otorgó subvenciones especiales para agilizar los proyectos en curso y multiplicar el número de publicaciones, contratar (nuevos) investigadores y mejorar las instalaciones tales subsidios "elefantiásicos" procedieron de aportes externos a la Facultad, como la ICE y el Congreso Nacional-; 2) la crisis de la ciencia española, en virtud de la cual el Instituto ganó un lugar dentro de la filología hispánica y se erigió como digno heredero del $\mathrm{CEH}$, "a contrapelo de" la coyuntura internacional; y finalmente, el Instituto se convirtió en un centro neurálgico del campo disciplinar en Hispanoamérica e ingresó al seno de la academia norteamericana al establecer intercambios con Estados Unidos. Por esta razón, Lida considera que el posicionamiento internacional alcanzado por el Instituto durante la década de 1930 no puede ser únicamente atribuido al "talento" de Alonso y su labor desarrollada en Buenos Aires, sino a "la coyuntura internacional, que hizo que la investigación lingüística se viera perjudicada en otras latitudes", principalmente en la Península, a raíz del ascenso de Franco y del colapso del CEH.

El capítulo cuarto se detiene en las vidas literaria y pública del Instituto, que, a criterio de la autora, supo hacerse un lugar en la escena cultural y política porteña gracias a la polifacética y empática personalidad de su director. Constituyen firmes testimonios de ello los vínculos con diferentes revistas de la arena intelectual argentina: Nosotros (1907-1943) -fundada por Alfredo Bianchi y Roberto Giusti-, que realizaba banquetes en honor a grandes figuras de las letras hispanoamericanas, y Sur (1931-1992) -fundada por Victoria Ocampo en 1931-, en la que se incorporaron al núcleo editor Henríquez Ureña y, como colaboradores invitados junto con otros de sus discípulos, Alonso y Alfonso Reyes. Esta situación de visibilidad pública y política le permitió al filólogo navarro establecer fuertes lazos con las elites de las principales entidades comunitarias de españoles instaladas en la Argentina -la ICE, la Asociación Patriótica, la Cámara Española de Comercio, etcétera-. En 1934, incluso, fue nombrado agregado cultural de la embajada de España en Buenos Aires, aunque dos años después debió renunciar a dicho cargo y nacionalizarse argentino para poder continuar su labor en la Universidad de Buenos Aires, porque el gobierno no permitía que los extranjeros ocuparan cátedras universitarias (135-138). Así, Lida caracteriza un nuevo posicionamiento de Alonso, quien se veía como un "excelente intermediario" entre España y la Argentina (eventualmente, Hispanoamérica): "una suerte de embajador cultural entre ambas geografías, sensibilidades, usos de la lengua, mercados y arenas culturales" (137), o bien un "articulador cultural, capaz de tender diálogos y de hacer comprensibles las plumas argentinas y latinoamericanas a las españolas y viceversa" (138). Hacia 1937, explica la autora, Alonso, desde Buenos Aires, se solidarizó con el exilio republicano y mostró su pleno compromiso con la Junta de Ayuda Argentina para los Universitarios Españoles, que contó con Bernardo Houssay como presidente y con Francisco Romero como secretario. En este contexto se estableció, entre otras -Sudamericana y Emecé, por ejemplo-, la principal casa editora del exilio español: Losada, sello que procuró alcanzar una producción de calidad en un libro accesible en costo que apuntaba a la industria cultural de masas, alentando publicaciones de obras especializadas y extendiendo su mirada a un público no académico con el que capitalizar el crecimiento económico de Buenos Aires. Alonso, justamente, se convirtió en asesor literario, traductor, autor, editor y director de las referidas colecciones de esta firma: CEE y CFyTL.

En este marco, explica Lida, Buenos Aires devino "meridiano intelectual de Hispanoamérica". El rol del filólogo no era el de "un inquisidor con aires de pedagogo"; no se mostraba como un predicador de normas, no velaba por la corrección lingüística, sino que con su tarea contribuía con la "nivelación idiomática”. El afianzamiento de un español neutro, despojado de localismos y particularismos de difícil comprensión para hablantes de otras latitudes, según Alonso, no obedecía a un criterio normativo, sino industrial (de índole cultural), pues permitiría a los productores (de libros y radios) alcanzar con mayor facilidad los mercados hispanoamericanos; su idea era que la capacidad de interpelación de estos materiales no se agotara en un lector y/o auditor argentino. Este era el enfoque con el que Alonso procuraba salvar la cuestión de la lengua en la Argentina, donde no había razones para someterse a "un estrecho nacionalismo lingüístico de tintes rioplatenses", pues en un mundo interconectado y global, explica Lida, "lo nacional quedaba reducido a mero localismo". La unidad cultural hispanoamericana, entonces, ya no contaba con un único centro (Madrid), sino que otros dos focos (Buenos Aires y México) “debían cooperar dignamente en la incesante formación de la lengua general”.

El quinto capítulo ofrece el desenlace de la gestión de Alonso a partir del "sacudón" que el golpe militar de 1943 propició al sistema universitario argentino, justo 
en un momento en el que el contexto internacional comenzó a recuperarse. En los últimos años, Alonso había fortalecido los estrechos contactos con la academia científica norteamericana; así fue que hacia 1946 recibió una invitación de la Universidad de Harvard, por la que pidió una licencia, pero los “yanquis" no estaban bien vistos por el gobierno peronista, de corte decididamente antinorteamericano, y Alonso fue entonces cesanteado por "desapego a la cultura argentina", hecho que, sumado a las constantes intervenciones estatales, generó entre los miembros del Instituto "un clima de desazón que rememoraba la destrucción del CEH".

Una vez instalado en Estados Unidos, explica la autora, Alonso consiguió "revivir lazos de camaradería con amigos de antaño", gozó de "una vida académica estable y no sujeta a vaivenes políticos y a restricciones presupuestarias", pero padeció "el sinsabor" de residir en "un país por lo demás ajeno a la comunidad hispánica”.

Lida concluye que, desplazado Alonso, del Instituto "solo quedaría una cáscara vacía" (166). Tal afirmación -incluso a la luz de la mejor de las interpretaciones- requeriría algún tipo de matiz - o al menos restricción temporal-, pensamos, en consideración del peso (intelectual) de muchas de las figuras que enriquecieron y gestionaron el organismo a lo largo de las décadas subsiguientes.

Un comentario final, a nuestro criterio, se impone. Como anticipamos, parafraseando a la autora, Amado Alonso en la Argentina (2019) tiene una fuerte intertextualidad con Años dorados de la cultura argentina (2014). En algunos capítulos hay, lógicamente, parciales recurrencias de contenidos y formulaciones, pero sobre todo importa destacar la persistencia de una idea rectora que vertebra ambas obras: la relevancia del contexto, de las circunstancias y condiciones históricas, políticas y culturales en el destino de las personas y de las instituciones. El libro de 2014 acomete en contra de la imagen que el nombre "década infame" construyó y solidificó, puesto que ella oculta aspectos muy significativos y positivos de dicho período. La metáfora suele encontrar lugar privilegiado en la argamasa de la denominación política; dada su naturaleza analógica-se caracteriza por destacar algunos rasgos de significado y, simultáneamente, ocultar o postergar otros- suele condicionar la percepción y la inferencia: esa década, argumenta Lida, no solo fue una época de "fraude patriótico, avanzada militar y clerical", sino que también recogió los frutos de la democratización política derivada de la ley Sáenz Peña, que hizo posible la movilización cultural de las clases medias y alcanzó tal vitalidad que convirtió a Buenos Aires en un centro de gran efervescencia intelectual. Ese contexto socio-histórico y cultural permitió, habilitó y condicionó el período áureo del Instituto de Filología, el formidable éxito de Alonso en sus múltiples facetas y la formación de excelencia en investigación de los discípulos: entre ellos, Ángel Rosenblat, Berta Vidal de Battini y, por supuesto, los hermanos Raimundo y María Rosa Lida.

Una diferencia importante de aquel libro con el que aquí comentamos radica en el punto de vista de la elaboración. Amado Alonso en la Argentina es una historia global del Instituto, pensada desde el otro lado del Atlántico gracias a una estadía en la Universidad de Cantabria (España), que le permitió a la autora complementar archivos americanos de distintas procedencias -incluido el del Instituto de Filología- con diferentes repositorios españoles, y también dialogar con especialistas de aquella geografía. Aventuramos que esta diferente perspectiva pueda haber iluminado (en parte) una formulación de base metafórica recurrente en el libro, que forma parte principal de la tesis central: "a contrapelo" -por ejemplo, la primera vez en la página 18, luego en la 96 y, por último, en la 163-. Una idea defendida por la autora -tal vez la hipótesis principal que caracteriza acertadamente como contrafactual- es que los logros del Instituto, su época dorada, fueron "tanto más luminosos gracias a lo sombrío del panorama internacional circundante" y, más adelante, aparece la frase focalizada: "la época dorada del Instituto del Filología de Buenos Aires fue a contrapelo, en un momento en que se había quedado sin competencia por la fuerza de las circunstancias, lo cual le permitió descollar...". Los diccionarios dicen: $a$ contrapelo: fig. "contra el curso o modo natural de una cosa" (RAE, 2014); "se usa para indicar que se realiza una acción de manera contraria a la usual o admitida en la expresión a contrapelo" (Larousse, 2016). Lida recurre con frecuencia a las metáforas para presentar valoraciones, que son los lugares del texto que, no casualmente, provocan y estimulan la polémica. En el libro, a nuestro entender, pueden percibirse ciertas tensiones en la exposición, que están anunciadas en la introducción, y que se proyectan en los distintos capítulos. Una de las principales es, justamente, el peso que habría tenido el contexto (global y local) en el éxito inusitado del Instituto y el papel de Amado Alonso. La autora, como anunciamos, no tiene voluntad hagiográfica, pero a pesar de no encorsetarse en la biografía, no puede evitarla, puesto que, como sostiene: "Alonso fue su protagonista, si bien en circunstancias que él mismo no creó en la mayoría de los casos. En pocas palabras, el Instituto de Filología argentino y todo su prestigio fueron frutos de su tiempo". 
No obstante, la lectura de los capítulos centrales que documentan con rigurosidad y nutrido sustento testimonial la descomunal obra de Alonso en y desde el Instituto va mitigando esa afirmación algo contundente. Desde la llegada del joven navarro al centro de investigación porteño, la descripción de la historia del Instituto aumenta el protagonismo de su figura: su capacidad de adaptación a la sociedad argentina, el magnetismo de su personalidad, el carácter llano y sociable, su sensibilidad y sagacidad como fonetista, su talento, su inteligencia y su profusa obra científica, editorial y cultural, su habilidad para gestionar fondos y para tejer redes de cooperación interinstitucionales e internacionales fueron, evidentemente, una pieza esencial en el éxito del Instituto. No hay hagiografía, pero la descripción determina, como efecto de lectura, la inferencia laudatoria; hay, incluso, cierta actitud crítica cuando en distintos pasajes se insinúa el "caciquismo varonil" propio de los directores de instituto de la época, del que Alonso no habría escapado, aunque también este reparo encuentre su morigeración cuando la autora documenta el apoyo "sin cortapisas" a las carreras de las investigadoras mujeres, como María Rosa Lida, y el haberse rodeado de talentosos discípulos judíos, a quienes promovió y apoyó a contracorriente en tiempos de nacionalismos católicos.

¿Fueron el Instituto de Filología y todo su prestigio frutos de su tiempo? A medida que progresa la obra esa tesis encuentra, en nuestra opinión, una formulación más ajustada que resuelve aquella tensión. En el capítulo cuarto, Miranda escribe:

Así, pues si el Instituto de Filología de Buenos Aires, encabezado por su director, pudo llevar adelante una marcha impetuosa que le permitió trascender fronteras y alcanzar cuotas de prestigio, ello se debe no solo al talento individual de su director y al trabajo de todo el equipo que lo acompañaba, sino también a la existencia de un fructífero contexto cultural que le resultó estimulante para toda su producción (127)

De este modo, se acerca a una visión bastante compartida en la comunidad disciplinar, que es la que ofreció Eugenio Coseriu en 1952 apenas enterado de la muerte de Alonso: fue en una conferencia que caracterizó como “cuasi-improvisación” y que fue pronunciada en una sesión extraordinaria del Centro de Lingüística de Montevideo (Uruguay), convocada para conmemorar la figura del fallecido. El fragmento es prolífico en metáforas -no del todo celebrables, por cierto-y apela, como Lida en ocasiones, a la contrafactualidad:
No hay duda, pues, que aun si hubiese quedado en España, Amado Alonso habría llegado a ser un gran profesor y un estudioso insigne. Pero, trasladado en 1927 a América -propuesto por Menéndez Pidal para dirigir el Instituto de Filología de Buenos Aires-, fue mucho más que eso: fue realmente el conquistador y organizador de nuevas e inmensas tierras para el dominio de la glotología hispánica y de la ciencia lingüística en general y, a pesar de su juventud (o, mejor quizás, justamente gracias a ella), supo ser lo que, con una expresión que ya se ha vuelto trivial, se dice "the right man in the right place" (Coseriu, 1952: 34).

En definitiva, se trató de un caso de condicionamiento recíproco y afortunado entre contexto y personaje. Azar y voluntad, o bien determinación y golpe de suerte -tal como sentenciaba Guitarte en el fragmento que colocamos de epígrafe para la presente reseña-, elementos de naturaleza dispar que asignaron coordenadas espacio-temporales al sujeto (Alonso) y al organismo (el Instituto) para que cada uno llegara a ser lo que fue. Justamente, "Llega a ser el que eres" (1929), predicaba el joven Alonso (siguiendo la recomendación de Platón), cuando consideraba que ese era "el único sentido vitalmente decoroso para el hispano-americanismo" (33).

El libro de Miranda Lida es, para finalizar, una obra de referencia para la comprensión de una etapa emblemática de la cultura argentina, de la filología y la lingüística en lengua castellana y, por supuesto, de la Universidad de Buenos Aires y de nuestro Instituto de Filología. Creemos que logra soberanamente su objetivo: presentar y condensar de manera elocuente el impacto que el contexto histórico-político ejerce sobre las posibilidades que, como sociedad y como individuos, tenemos para trabajar, lograr, proyectar y brindar a los demás.

\section{Bibliografía}

Alonso, A. (1929). “Llega a ser el que eres”. La Nación, 21 de julio, 32-33.

Coseriu, E. (1952 [1996]). “Amado Alonso (18961952)". Lexis, XX, 1-2, 31-41.

Diccionario Larousse (2016). Disponible en: https:// www.diccionarios.com

Guitarte, G. (1952). “Amado Alonso”. Filología, IV, 3-7. 
Lida, M. (2013). Monseñor Miguel De Andrea. Obispo y hombre de mundo. Buenos Aires: Edhasa.

Lida, M. (2014). Años dorados de la cultura. Los hermanos María Rosa y Raimundo Lida y el Instituto de Filología antes del peronismo. Buenos Aires: EUDEBA.

Lida, M. (2015). Historia del catolicismo en la Argentina. Entre el siglo XIX y el XX. Buenos Aires: Siglo XXI.
Lida, M. (2019). Amado Alonso en la Argentina. Una historia global del Instituto de Filología (1927-1946). Bernal: Universidad Nacional de Quilmes.

Real Academia Española (2014). Diccionario de la Lengua Española. Madrid: Espasa Calpe, 23를 edición. Disponible en: https://dle.rae.es/ 